\title{
Prevalencia y posibles factores de riesgo en la excreción de ooquistes de Cryptosporidium spp en bovinos de Boyacá, Colombia
}

\author{
Prevalence and possible risk factors for \\ Cryptosporidium spp oocyst excretion in dairy \\ cattle in Boyacá, Colombia
}

\author{
Martín Orlando Pulido-Medellína, Roy José Andrade-Becerraa, Roger Iván Rodríguez- \\ Vivasb, Diego José Garcia-Corredora
}

\begin{abstract}
RESUMEN
El objetivo fue determinar la prevalencia y posibles factores de riesgo asociados a la excreción de ooquistes de Cryptosporidium spp en excremento de bovinos Holstein en fincas de producción lechera de Boyacá, Colombia. Se colectaron 100 muestras de materia fecal de bovinos hembras mediante estimulación rectal. Con la técnica de Ziehl Neelsen modificada se determinó la excreción de ooquistes de Cryptosporidium spp. Para conocer los posibles factores asociados se usó la prueba de Ji cuadrada teniendo como variable dependiente la presencia de ooquistes de Cryptosporidium spp y como independientes la presencia de diarrea (si, no), edad ( $<12$ meses, $>12$ meses), finca de procedencia $(1,2,3,4)$ y estado reproductivo (gestante, vacía). Las variables con $P<0,05$ fueron analizadas mediante regresión logística. La prevalencia general de bovinos excretando ooquistes de Cryptosporidium spp fue de $48 \%$ (48/ 100). Las explotaciones ganaderas estudiadas presentaron prevalencias de $11.1,43.8,50.8$ y $66.6 \%$ para las explotaciones 1, 2, 3 y 4 respectivamente $(P>0.05)$. Las variables que durante el análisis con $X^{2}$ tuvieron valores $P<0.05$ fueron edad y presencia de diarrea. Los bovinos con diarrea $(O R=2.99, P=0.038)$ y $<12$ meses de edad $(0 R=$ 9.42, $P=0.037$ ) fueron los factores asociados a la excreción de ooquistes de Cryptosporidium spp. Los bovinos de fincas lecheras de Boyacá, presentan alta prevalencia de excreción de ooquistes de Cryptosporidium spp; bovinos con diarrea y menores de $\mathbf{1 2}$ meses de edad son los que presentaron el parásito con mayor frecuencia.
\end{abstract}

PALABRAS CLAVE: Cryptosporidium spp, Protozoario, Enteropatías, Ooquistes, Factores de riesgo.

\begin{abstract}
The objective was determined the prevalence and potential risk factors associated to the excretion of Cryptosporidium spp oocyst in Holstein cattle from dairy farms in Boyaca, Colombia. One hundred fecal samples were taken from females cattle by rectal stimulation. The excretion of Cryptosporidium spp oocyst was determined by the modified Ziehl Neelsen technique. To determine the potential associated factors, the $\mathbf{C h i}^{2}\left(\mathrm{x}^{2}\right)$ test was used, having as dependent variable the excretion of Cryptosporidium spp oocysts, and as independent variables the presence of diarrhea (yes, no), age (<12 mo, $>12 \mathrm{mo})$, localization $(1,2,3,4)$, and reproductive status (pregnant, non pregnant). Variables with $\boldsymbol{P}<0,05$ were analyzed by logistic regression. The general prevalence of cattle excreting Cryptosporidium spp oocyts was $48 \%(48 / 100)$. The farms studied presented prevalence of $11.1,43.8,50.8$, and $66.6 \%$ for farms $1,2,3$ and 4 respectively $(P>0.05)$. Variables that showed $P<0.05$ in the $x^{2}$ analysis were age and presence of diarrhea. Cattle with diarrhea $(O R=2.99, P=0.038)$, and $<12$ mo of age $(O R=9.42, P=0.037)$ were associated with the excretion of Cryptosporidium spp oocysts. Cattle from dairy farms in Boyacá, present a high prevalence of Cryptosporidium spp oocyst excretion; cattle with diarrhea and minors of 12 mo of age are those who present the parasite with major frequency.
\end{abstract}

KEY WORDS: Cryptosporidium spp, Protozoo, Enteropathy, Oocyst, Risk factors.

Recibido el 29 de enero de 2013. Aceptado el 11 de marzo de 2013.

a Grupo de Investigación GIDIMEVETZ, Programa de MVZ Universidad Pedagógica y Tecnológica de Colombia. Colombia. mopm1@hotmail.com. Correspondencia al primer autor.

b Facultad de Medicina Veterinaria. Universidad Autónoma de Yucatán. México. 
La criptosporidiosis es una enfermedad causada por un protozoario del género Cryptosporidium (Apicomplexa: Cryptosporiidae) que puede afectar a diversas especies, incluyendo vertebrados e invertebrados. La enfermedad se caracteriza por la presentación de cuadros diarreicos debido a que afecta principalmente las células epiteliales del tracto gastrointestinal. El género Cryptosporidium se diferencia de otros coccidios, ya que todas las etapas de su ciclo vital ocurren dentro de un solo huésped y además se desarrolla dentro de la célula epitelial, pero en una vacuola extra-citoplasmática. Afecta principalmente a la especie bovina y los datos sobre prevalencia muestran variaciones que se relacionan con las condiciones epidemiológicas, la zona geográfica estudiada, la historia clínica del hato, el sistema de explotación, las prácticas de higiene, el manejo y la edad al momento del muestreo de los bovinos(1).

Actualmente, al menos 13 especies así como varios genotipos han sido reconocidos en el género Cryptosporidium empleando principalmente criterios morfológicos, moleculares y especificidad de hospederos $(2,3)$. En el ganado bovino se han reconocido dos especies, Cryptosporidium spp que infecta el intestino de becerros neonatos, y Cryptosporidium andersoni, que infecta el abomaso de animales jóvenes y adultos(3). En los últimos años se han identificado también dos genotipos, denominados Bovino B, actualmente C. bovis, y "deer-like", de igual manera, se han registrado numerosos subgenotipos de Cryptosporidium $\operatorname{spp}(4,5)$.

El principal mecanismo de transmisión de Cryptosporidium spp es la vía oral-fecal, ya que los ooquistes son encontrados exclusivamente en el excremento, que generalmente contamina agua, suelo, forrajes, comederos, etc.; facilitando así la diseminación de los ooquistes, que son infectivos desde el momento de su excreción(6).

Dentro de la especie bovina, los becerros son especialmente susceptibles a la infección con
Cryptosporidiosis is caused by a Cryptosporidium (Apicomplexa: Cryptosporiidae) genus protozoan and can occur in numerous vertebrate and invertebrate animal species. It primarily affects gastrointestinal epithelial cells, and is characterized by diarrhea. Unlike other coccidia, Cryptosporidium genus protozoa can complete all their lifecycle stages in a single host, and develop inside the epithelial cell within an extracytoplasmic vacuole. The disease mainly affects cattle, with prevalence data varying in response to epidemiological conditions, geographic zone, herd clinical history, production system hygiene and management practices, and age at sampling(1).

At least thirteen species and a number of genotypes are currently identified within the Cryptosporidium genus, based mainly on morphological, molecular and host specificity criteria(2,3). Two species have been identified in cattle, Cryptosporidium spp, which infects the intestine of newborn calves, and Cryptosporidium andersoni, which infects the abomasum in young and adult animals(3). Two additional genotypes have been identified in recent years: Bovine $B$, currently referred to as C. bovis; and another known as "deer-like". In addition, a number of Cryptosporidium spp subgenotypes have been recorded $(4,5)$.

Cryptosporidium spp is transmitted via the oralfecal route since oocysts are present only in feces and are infectious from the moment of excretion(6). They then contaminate water, soil, forage, feeders, etc., facilitating oocyst dissemination.

In cattle, calves are especially susceptible to Cryptosporidium spp infection. Calves as young as $2 \mathrm{~d}$ can be infected, although prevalence is greater between 15 and $21 \mathrm{~d}$ of age. Most cases in calves exhibit parasite-origin diarrhea. Adults normally develop an active but asymptomatic infection, excreting large quantities of oocysts and efficiently perpetuating infection within production units(7). Diarrhea in calves is the main cause of morbidity and 
Cryptosporidium spp. Aunque se ha demostrado la infección desde los dos días de nacidos, la mayor prevalencia ocurre en becerros de 15 a 21 días de edad, los cuales en la mayoría de los casos presentan cuadros diarreicos ocasionados por este parásito; en contraste, los animales adultos, desarrollan infección activa del parásito y generalmente son asintomáticos, pero excretan gran cantidad de ooquistes, perpetuando la presencia de la enfermedad al interior de las explotaciones(7). Debido a que la causa principal de morbilidad y mortalidad en terneros es la diarrea, la criptosporidiosis en los primeros días de vida es el proceso patogénico más importante de los producidos por parásitos en el ganado bovino(8).

Cryptosporidium spp ha llamado el interés de muchos investigadores en el mundo, que reportan diversos estudios de prevalencia, subtipificación y análisis filogenético, rutas y fuentes de transmisión, inmunología, métodos diagnósticos, tratamientos y estudios farmacológicos, repercusiones en la salud pública, entre otros.

La prevalencia de bovinos positivos a Cryptosporidium spp es variable dependiendo del país y las condiciones particulares de cada región. En Europa los reportes de prevalencias varían entre 15.6 y $83 \%(9,10)$. En América las prevalencias presentan variaciones que van desde 9.7 hasta $75 \%(11,12)$. En Colombia existen pocos estudios, entre ellos uno realizado en el departamento de Cundinamarca, donde se estudiaron 135 muestras de sangre bovina de siete explotaciones ganaderas para determinar la seroprevalencia a Cryptosporidium spp, encontrándose el $53.3 \%$ de reactores positivos(7).

Por su carácter zoonótico, Cryptosporidium spp representa un riesgo potencial en la trasmisión hacia los bovinos y otras especies animales, incluyendo a los humanos(13). Debido a la importancia que adquiere Cryptosporidium spp, como responsable de la mayoría de enteropatías causadas por protozoarios en bovinos y en diversas especies, y al desconocimiento de su mortality at this stage, making cryptosporidiosis in the first days of life the most important parasite-origin pathogenic process in cattle ${ }^{(8)}$.

Cryptosporidium spp has received extensive research interest worldwide resulting in various studies on prevalence, subtypification and phylogenetic analysis, transmission sources and routes, immunology, diagnostic methods, treatment, pharmacology and public health repercussions, among others.

Prevalence among cattle varies by country and regional conditions. In Europe, it ranges from 15.6 to $83 \%(9,10)$, while in the Western Hemisphere it ranges from 9.7 to $75 \%(11,12)$. Among the few studies addressing Cryptosporidium spp in Colombia is one done in Cundinamarca department in which $53.3 \%$ of 135 cattle blood samples from seven production units were positive reactive(7). Cryptosporidium spp is the main cause of protozoan-caused enteropathies in cattle and other species (including humans) because it is a zoonotic agent and therefore poses a serious potential transmission risk(13).

No data is available on Cryptosporidium spp prevalence in dairy cattle ranches in Boyacá department, Colombia. The present study objective was to determine Cryptosporidium spp prevalence, and identify risk factors associated with oocyst excretion in Holstein feces in Boyacá.

Boyacá department, Colombia is located at $7^{\circ}$ $08^{\prime} \mathrm{N}, 4^{\circ} 39^{\prime} \mathrm{N}, 71^{\circ} 56^{\prime} \mathrm{W} 74^{\circ} 38^{\prime} \mathrm{W}$; a region with a cold climate (average annual temperature of $\left.16^{\circ} \mathrm{C}\right)(14)$. All the cows at four ranches $(1$, 2,3 and 4) were sampled, resulting in 100 fecal samples (9 from ranch 1, 16 from ranch 2, 63 from ranch 3, and 12 from ranch 4). In animals older than $6 \mathrm{mo}$, samples were collected by inserting the forearm, covered with a disposable glove, directly into the rectum and extracting feces. In animals less than 6 mo of age, two fingers were used to stimulate the rectum, and feces collected as it was excreted. 
prevalencia dentro de las explotaciones de producción lechera del departamento de Boyacá, el presente estudio tuvo como objetivo determinar la prevalencia y factores de riesgo asociados a la excreción de ooquistes de Cryptosporidium spp en excremento de bovinos Holstein en explotaciones de producción lechera de Boyacá, Colombia, ubicado en el norte a los $7^{\circ} 08^{\prime} \mathrm{N}$, y al sur $4^{\circ} 39^{\prime} \mathrm{N}$. Por longitud, al oriente, $71^{\circ} 56^{\prime} \mathrm{O}$ de Greenwich, y al occidente $74^{\circ} 38^{\prime} \mathrm{O}$; es una región de clima frío con temperatura promedio de $16^{\circ} \mathrm{C}(14)$.

Se seleccionaron cuatro explotaciones, donde se muestrearon todas las hembras de cada finca, colectándose un total de 100 muestras de materia fecal, directamente del recto de los animales con mangas desechables estériles; introduciendo el antebrazo en individuos mayores de seis meses, e introduciendo dos dedos para estimular el recto en individuos menores de seis meses. Las muestras fueron inmediatamente transferidas a una cámara fría portátil para ser transportadas al Laboratorio de la Clínica Veterinaria de pequeños y grandes animales de la Universidad Pedagógica y Tecnológica de Colombia, donde se procesaron de inmediato para evitar que los ooquistes sufrieran alteraciones que pudiesen dificultar su identificación. De cada animal muestreado se obtuvo la siguiente información: identificación, edad, estado reproductivo, presencia de diarrea y nombre de la explotación.

El número de muestras obtenidas fueron 9,16 , 63 y 12 para las fincas $1,2,3$ y 4 respectivamente. Para el diagnóstico de Cryptosporidium spp se usó la técnica de tinción de Ziehl Neelsen modificada descrita por González y Moreno, la cual presenta una sensibilidad de $88 \%$ y especificidad de $92 \%(15)$. La muestra se observó al microscopio óptico para determinar la presencia de ooquistes de Cryptosporidium spp utilizando el objetivo 100x.

Como variable dependiente se consideró la presencia de ooquistes de Cryptosporidium spp, y como variables independientes: presencia de
Samples were immediately placed in a portable cold room for transport to the Small and Large Animal Veterinary Clinic, Teaching and Technological University of Colombia (Universidad Pedagógica y Tecnológica de Colombia). Once in the laboratory, samples were processed immediately to avoid any alteration of oocysts that could affect identification. For each sampled animal, data was collected on ranch name, identification number, age, reproductive condition, and presence or absence of diarrhea.

Cryptosporidium spp diagnosis was done using the Ziehl-Neelsen stain technique, with modifications, a technique with $88 \%$ sensitivity and $92 \%$ specificity(15). Oocysts were identified with a microscope (100x).

Oocyst presence was the dependent variable. Four independent variables were included: presence of diarrhea (yes, no); age ( $<12 \mathrm{mo}$, $>12 \mathrm{mo})$; ranch $(1,2,3,4)$; and reproductive condition (pregnant, open).

Risk factors associated with Cryptosporidium spp oocyst excretion were evaluated with a univariate analysis using a $\mathrm{Chi}^{2}$ test. Variables with values of $P<0.05$ were analyzed with a multivariate logistical regression (Statistics ver. 9 software) to calculate the odds ratio (OR). Apparent (AP) and real prevalences (RP) were calculated using the formulas(16):

$A P=$ (No. Positive animals / total population) $\mathrm{x} 100$.

$\mathrm{RP}=\left(\mathrm{AP}+\right.$ test $\left.^{\text {specificity }}{ }^{-1}\right) /$ (test sensitivity $^{-1}$ + test specificity $^{-1}$ )

Overall RP of Cryptosporidium spp oocysts in excreta was $48 \%$ (48/100), with specific rates of $11.1 \%(1 / 9)$ at ranch $1,43.8 \%(7 / 16)$ at ranch $2,50.8 \%(32 / 63)$ at ranch 3 , and $66.6 \%$ $(8 / 12)$ at ranch $4(P>0.05)$. In the $\mathrm{Chi}^{2}$ univariate analysis, age results exhibited significantly different values in which $90.9 \%$ of young $(<12 \mathrm{mo})$ animals were positive for oocysts and were 9.42 times more likely to excrete oocysts. In addition, $70.4 \%$ of those 
diarrea (si, no), edad ( $<12$ meses, $>12$ meses), finca de procedencia $(1,2,3,4)$ y estado reproductivo (gestante, vacía).

Para determinar los factores de riesgo asociados a la excreción de Cryptosporidium spp se realizó un análisis univariado mediante la prueba de $\mathrm{Ji}^{2}$. Las variables con valores de $P<0.05$ fueron analizados mediante la prueba multivariada de regresión logística (Statistics software, versión 9) para obtener la razón de momios (valor de $\mathrm{OR})$.

Para determinar las prevalencias aparente y real se utilizaron las fórmulas descritas por Altman y Bland(16):

Prevalencia aparente: (No. animales positivos / población total) x 100 .

Prevalencia real: (prevalencia aparente + especificidad de la prueba ${ }^{-1}$ ) / (sensibilidad de la prueba + especificidad de la prueba- ${ }^{-1}$ ).

La prevalencia real de bovinos con excreción de ooquistes de Cryptosporidium spp fue de $48 \%$ (48/100). Las cuatro explotaciones estudiadas presentaron prevalencias de $11.1 \%$ (1/9), $43.8 \%$ (7/16), $50.8 \%$ (32/63), $66.6 \%$ $(8 / 12)(P>0.05)$. animals with diarrhea had Cryptosporidium spp oocysts in their excreta, while only $39.7 \%$ of those without diarrhea had oocysts. Both of these variables were significant in the logistical regression analysis, with OR values of 9.42 for age $<12 \mathrm{mo}$ and 2.99 for diarrhea (Table 1).

The $48 \%$ RP for Cryptosporidium oocyst excretion in the studied population was similar to the $53.3 \%$ reported in a serological study of 135 cows in seven dairy ranches in Cindinamarca department, Colombia(7), and comparable to the $47.8 \%$ prevalence reported in northern Táchira State, Venezuela(15). However, the overall RP prevalence observed in the present study was quite different from the $4.9 \%$ reported in a study indicating that good management practices and knowledge of Cryptosporidium spp epidemiological behavior are fundamental to controlling cryptosporidiosis.

Cryptosporidium oocyst excretion prevalence varies worldwide depending in climate conditions, animal age and production system hygiene management. In Europe, prevalence ranges from 15 to $83 \%(8,9,10,13,18,19)$, Spain having the highest prevalence in cattle, while in the Americas prevalence varies from 10 to $75 \%(11,12,15,20-23)$.

Cuadro1. Factores asociados a la excreción de ooquistes de Cryptosporidium spp en heces de bovinos de Boyacá, Colombia

Table 1. Factors associated with Cryptosporidium spp oocyst excretion in feces in dairy cows in Boyaca, Colombia

\begin{tabular}{lcccccc}
\hline Variable & No. of animals & Positives & $\%$ Infection & OR & $95 \% \mathrm{Cl}$ & $P$ \\
\hline Diarrhea & 73 & 29 & 39.7 & & & \\
No & 27 & 19 & 70.4 & 2.99 & $113-7.86$ & 0.038 \\
Yes & & & & & & \\
Age & 89 & 38 & 42.7 & & & \\
$\geq 12$ mo & 11 & 10 & 90.9 & 9.42 & $1.14-78.12$ & 0.037 \\
$<12$ mo & & & & & & \\
Reproductive stage & 57 & 33 & 57.8 & & & \\
Open & 43 & 15 & 34.8 & 0.74 & $0.31-1.18$ & 0.500 \\
Pregnant & &
\end{tabular}

$\mathrm{Cl}=$ Confidence interval; $\mathrm{OR}=$ Odds ratio; $\mathrm{P}=$ probability value. 
Las variables que en el análisis univariado de $\mathrm{Ji}^{2}$ tuvieron valores con $P<0.05$ fueron edad, donde se encontró que el $90.9 \%$ de los animales jóvenes ( $<12$ meses) resultaron positivos a Cryptosporidium spp y tuvieron 9.42 veces más probabilidad de excretar ooquistes; y presencia de diarrea donde el $70.4 \%$ de los animales con diarrea presentó excreción de ooquistes de Cryptosporidium spp, mientras que sólo el $39.7 \%$ de los animales no diarreicos presentó excreción de ooquistes.

En el Cuadro 1 se presentan los resultados de la regresión logística de las variables con $P<0.05$. Las variables presencia de diarrea $y$ edad $<12$ meses fueron significativas con valores OR de 2.99 y 9.42 respectivamente.

La prevalencia real de bovinos con excreción de ooquistes de Cryptosporidium spp fue de $48 \%$; esta prevalencia es similar a la obtenida en un estudio serológico realizado en el departamento de Cundinamarca, Colombia (53.3 \%) de 135 bovinos en siete explotaciones lecheras(7); además es comparable con la prevalencia de $47.8 \%$ obtenida en un estudio similar realizado en la zona norte del estado de Táchira, Venezuela(15). Sin embargo, la prevalencia obtenida se aleja de los resultados registrados por Hernández y Cortés(17), quienes determinaron la presencia de Cryptosporidium spp en el $4.9 \%$ de las muestras analizadas, evidenciando que las buenas prácticas ganaderas y el conocimiento del comportamiento epidemiológico del parásito juegan un papel fundamental dentro del control de esta enfermedad.

La prevalencia de excreción de ooquistes de Cryptosporidium spp en distintas partes del mundo es variable de acuerdo a las condiciones climáticas, edad y manejo sanitario de la explotación. En Europa la prevalencia varía de 15 a $83 \%(8,9,10,13,18,19)$, siendo España uno de los países con mayor presencia de bovinos con Cryptosporidium spp. En América la prevalencia varía de 10 a $75 \%(11,12,15,20-23)$.
All four sampled ranches had at least some cows positive for oocysts, probably due to similar management practices. This level of ubiquity was also reported in a study done in Zambia using molecular and serological techniques(24). Clearly, Cryptosporidium spp is a worldwide animal health challenge.

Most of the animals with diarrhea were also positive for Cryptosporidium oocysts, and the statistical association analysis showed that animals with diarrhea were more likely $(P<0.05)$ to excrete oocysts than those without diarrhea. A significant association between diarrhea and Cryptosporidium infection at dairy ranches has been reported previously(24). Animals with diarrhea therefore represent a high transmission risk, particularly for calves and immunodepressed individuals(19).

In contrast, only $15.3 \%$ of the animals negative for Cryptosporidium had diarrhea, which can be attributed to other etiological agents. For instance, high prevalences of Eimeria spp, Strongyloides and Strongylids were found to cause diarrhea in cows in a study in Táchira State, Venezuela(15).

Many more young ( $<12 \mathrm{mo}$ ) animals were positive for Cryptosporidium than older animals (>12 mo); young animals were also 9.42 more likely to excrete oocysts. This indicates that calves have a higher probability of infection after birth. The primary infection vector is their mothers because they remain with their mothers for 48 to $72 \mathrm{~h}$ after birth, the newborn calves' immune system is immature and the parasite's life cycle involves a short prepatent period(20). Most (58.3\%) of the older (>12 mo) animals were negative for Cryptosporidium oocyst excretion. This coincides with a previous study showing that cows older than 12 mo of age have a strong immunological response, allowing them to control the parasite and lowering oocyst excretion prevalences(25). In a study done in Germany of Cryptosporidium spp subtypification and molecular characterization, this parasite was found to be the most prevalent in weaned calves 
Todas las explotaciones estudiadas presentaron bovinos con presencia de ooquistes de Cryptosporidium spp; situación que es similar a un estudio realizado en Zambia, utilizando técnicas diagnósticas moleculares y serológicas, donde se encontró el parásito en todas las explotaciones muestreadas(24), evidenciando con esto que la presencia de Cryptosporidium spp es un problema de sanidad animal común a nivel mundial. La prevalencia encontrada entre las cuatro fincas estudiadas posiblemente fue debido a condiciones similares de manejo.

El $70.4 \%$ de los animales con diarrea presentó excreción de ooquistes de Cryptosporidium spp, mientras que sólo el $39.7 \%$ de los animales no diarreicos presentó excreción de ooquistes. El análisis estadístico de asociación reveló que los animales diarreicos tienen más probabilidad de excretar ooquistes de Cryptosporidium spp en comparación con los animales no diarreicos $(P<0.05)$, resultado similar al estudio realizado por Geurden et al(24), donde encontraron una asociación significativa entre la presentación de diarrea y la infección por Cryptosporidium spp en granjas lecheras. Los animales que presentan diarrea representan un alto riesgo de transmisión de Cryptosporidium spp principalmente a los terneros y animales inmunodeprimidos(19).

Por otra parte, sólo el $15.3 \%$ de los animales negativos a Cryptosporidium spp presentaron diarrea, evento que se puede atribuir a otros agentes etiológicos como los encontrados en un estudio realizado en el estado de Táchira (Venezuela), donde se registró una alta prevalencia de Eimeria spp, Strongyloides y Strongilidos como agentes causales de diarrea en bovinos(15).

En cuanto a los grupos de edades se encontró que el $90.9 \%$ de los animales jóvenes ( $<12$ meses) resultaron positivos a Cryptosporidium spp y tuvieron 9.42 veces más probabilidad de excretar ooquistes, lo que indica que los animales tienen una mayor posibilidad de infección después del nacimiento, y que las madres desempeñan un papel fundamental and the agent responsible for $85 \%$ of infections, while in adult animals it caused only $1 \%$ of infections(19).

Cows at the sampled dairy farms in Boyacá, Columbia, had a high Cryptosporidium spp prevalence, which differed from most published figures in different countries. Risk factors can facilitate Cryptosporidium establishment in a dairy farm. Young animals ( $<12 \mathrm{mo}$ ) with diarrhea were found to more frequently harbor this parasite.

End of english version

como fuente de infección, ya que la mayoría de los becerros permanecen con su madre durante 48 a 72 h después del nacimiento; además de la inmadurez del estado inmunológico del neonato y el corto periodo prepatente del ciclo de vida del parásito(20). La mayoría de los animales mayores de 12 meses (58.3\%) fueron negativos a la excreción de Cryptosporidium spp, este hallazgo coincide con Fayer et al(25) quienes encontraron que los bovinos mayores a 12 meses presentan una respuesta inmunológica sólida, lo que les permite controlar al parásito y presentar menor prevalencia de excreción de ooquistes(25). En otro estudio realizado en Alemania donde se estudió la subtipificación y caracterización molecular de Cryptosporidium spp, se encontró que este parásito fue la especie más prevalente en terneros pre-destetetados y responsable del $85 \%$ de las infecciones en estos animales; mientras que en animales adultos sólo ocasionó el $1 \%$ de las infecciones(19).

Se concluye que los bovinos de las explotaciones lecheras de Boyacá, Colombia, presentan alta prevalencia de Cryptosporidium spp, y que los datos obtenidos difieren a las mayoría de cifras obtenidas durante investigaciones previas en diferentes países a nivel mundial. La presencia de factores de riesgo facilitan el establecimiento de Cryptosporidium spp al interior de fincas 
Martín Orlando Pulido-Medellín, et al. / Rev Mex Cienc Pecu 2014;5(3):357-364

lecheras; durante esta investigación se observó que bovinos con diarrea y menores de 12 meses de edad son los que con mayor frecuencia presentan el parásito.

\section{LITERATURA CITADA}

1. Venua R, Latha BR, Abdul-Basith SG, Dhinakar R, Sreekumar C, Ramana M. Molecular prevalence of Cryptosporidium spp in dairy calves in Southern states of India. Vet Parasitol 2012;188(2012):19-24.

2. Zhaoguo Ch, Rongsheng M, Huizhu $Y$, Yaojun Sh, Yan $H$, Yongjun Ch, Peng Z, Youmin C, Jiaojiao L. Prevalence of Cryptosporidium spp. in pigs in Shanghai, China. Vet Parasitol2011;181(2011):113-119.

3. Ramirez NE, Ward LA, Sreevatsan SA. Review of biology and epidemiology of cryptosporidiosis in humans and animals. Microbes Infect 2004;(6):773-785.

4. Slapeta J. Cryptosporidium species found in cattle: a proposal for a new species. Trends Parasitol 2006;(22):469-474.

5. Brook E, Hart CA, French N, Christley R. Prevalence and risk factors for Cryptosporidium spp infection in young calves. Vet Parasitol 2008;152:46-52.

6. Uga S, Matsuo J, Kono E, Kimura K, Inoue M, Rai SK. Prevalence of Cryptosporidium parvum infection and pattern of oocysts heedding in calves in Japan. Vet Parasitol 2007; 148:27-32.

7. Vergara-Castiblanco CA, Quílez-Cinca J, Freire-Santos F, Castro-Hermida JA, Ares-Mazás ME. Serological response to Cryptosporidium parvum in adult cattle from the Andean region of Colombia, J Parasitol 2004;120(3):243-248.

8. Silverlas C, Mattsson J, InsulanderM, LebbadM. Zoonotic transmission of Cryptosporidium meleagridis on an organic Swedish farm. Int J Parasitol 2012;42:963-967.

9. Cardona G, Carabin H, Goñi P, Arriola L, Robinson G, Fernández-Crespo J, Clavel A, Chalmers R, Carmena D. Identification and molecular characterization of Cryptosporidium and Giardia in children and cattle populations from the province of Álava, North of Spain. Sci Total Environ 2011;412-413:101-108.

10. Bendali F, Bichet $H$, Schelcher F, Sanaa M. Pattern of diarrhea in newborn beef calves in south-west France. Vet Res 2009;(30):61-74.

11. Ongerth J, Stibbs H. Prevalence of Cryptosporidium in dairy claves in Western Washington. Am J Vet Res 2009;(50):1069-1070.
12. Oliveira C. Occurrence of Cryptosporidium parvum in calf in the zone of Pereira, Municipality of Uberlandia- MG- Brasil. Int J Parasitol 2006;(13):590-593.

13. Wielinga $P$, De-Vries $A$, Van-Der-Goot $T$, Mank $T$, Mars $M$, Kortbeek L. Molecular epidemiology of Cryptosporidium in humans and cattle in The Netherlands. International J Parasitol 2008;(38):809-817.

14. Gobernación de Boyacá. Aspectos geográficos del departamento de Boyacá 2013. http://www.boyaca.gov.co/ ?idcategoria=3527. Consultado 24 ene, 2013.

15. González-Tapia L, Moreno-Manresa C, Identificación de patógenos causales de diarrea en terneros en la zona norte del estado Táchira. Universidad Nacional Experimental del Táchira (UNET) 2008. http://investigacion.unet.edu.ve. Consultado 14 ene, 2013.

16. Altman DG, Bland JM. Statistics Notes: Diagnostic tests 1: sensitivity and specificity. Br Med J 2001;(308):1552-1553.

17. Hernández-Gallo N, Cortés-Vecino JA. Prevalencia y factores de riesgo de Cryptosporidium spp. y Giardia spp. en terneros de ganado lechero de la zona noroccidental de la Sabana de Bogotá. Rev Salud Pública 2012;14(1):169-181.

18. Lentz T, Hofer D, Gottstei B, Gaillard C, Busato A. Prevalence and importance of endoparasites in calves raised in Swiss cow-calf farms. Deut Tierarztl Woch 2010;(106):275-281.

19. Broglia A, Reckinger S, Caccio SM, Nockler K. Distribution of Cryptosporidium parvum subtypes in calves in Germany. Vet Parasitol 2009;154(2009):8-13.

20. Trotz-Williams LA, Martin SW, Leslie KE, Duffield T, Nydam DV, Peregrine AS. Association between management practices and within-herd prevalence of Cryptosporidium parvum shedding on dairy farms in southern Ontario. Prev Vet Med 2008;(83):11-23.

21. Nevarez M, Ramírez R, Nino R, Rodríguez L. Identification of Cryptosporidium spp in calf with diarrhea. Clin Microbiol Rev 2009;(6):37-42.

22. Chirinos Y, Rojas M, Salinas G, Bastidas G, García F. Frecuencia de criptosporidiosis en becerreros de diez fincas de la zona ganadera de Tucacas, Estado Falcón, Venezuela. Rev Fac Cs Vets 2006;45(1):9-17.

23. Del-Coco VF, Córdoba MA, Basualdo JA. Cryptosporidium infection in calves from a rural area of Buenos Aires, Argentina. Vet Parasitol 2008;158:31-35.

24. Geurden T, Goma FY, Siwila J, Phiri IGK, Mwanza AM, Gabriel S. Prevalence and genotyping of Cryptosporidium in three cattle husbandry systems in Zambia. Vet Parasitol 2010;138:217-222.

25. Fayer R, Santín M, Trout J, Greiner E. Prevalence of species and genotypes of Cryptosporidium found in 1-2year-old dairy cattle in the eastern United States. Vet Parasitol 2010;135:105-112. 